\title{
Global format for energy-momentum based time integration in nonlinear dynamics
}

\section{Krenk, Steen}

Published in:

International Journal for Numerical Methods in Engineering

Link to article, DOI:

$10.1002 / \mathrm{nme} .4745$

Publication date:

2014

Link back to DTU Orbit

Citation (APA):

Krenk, S. (2014). Global format for energy-momentum based time integration in nonlinear dynamics.

International Journal for Numerical Methods in Engineering, 100(6), 458-476. https://doi.org/10.1002/nme.4745

\section{General rights}

Copyright and moral rights for the publications made accessible in the public portal are retained by the authors and/or other copyright owners and it is a condition of accessing publications that users recognise and abide by the legal requirements associated with these rights.

- Users may download and print one copy of any publication from the public portal for the purpose of private study or research.

- You may not further distribute the material or use it for any profit-making activity or commercial gain

- You may freely distribute the URL identifying the publication in the public portal

If you believe that this document breaches copyright please contact us providing details, and we will remove access to the work immediately and investigate your claim. 


\title{
Global Format for Energy-Momentum based Time Integration in Nonlinear Dynamics
}

\author{
Steen Krenk \\ Department of Mechanical Engineering Technical University of Denmark, Lyngby, Denmark
}

\begin{abstract}
SUMMARY
A global format is developed for momentum and energy consistent time integration of second order dynamic systems with general non-linear stiffness. The algorithm is formulated by integrating the state space equations of motion over the time increment. The internal force is first represented in fourth order form consisting of the end-point mean value plus a term containing the stiffness matrix increment. This form gives energy conservation for systems with internal energy as a quartic function of the displacement components. This representation is then extended to general energy conservation via a discrete gradient representation. The present procedure works directly with the internal force and the stiffness matrix at the time integration interval end-points, and in contrast to previous energy conserving algorithms does not require any special form of the energy function nor use of mean-value products at the element level or explicit use of a geometric stiffness matrix. An optional monotonic algorithmic damping, increasing with response frequency, is developed in terms of a single damping parameter. In the solution procedure the velocity is eliminated and the non-linear iterations are based on the displacement components alone. The procedure represents an energy consistent alternative to available collocation methods, with an equally simple implementation. Copyright (c) 2014 John Wiley \& Sons, Ltd.
\end{abstract}

Received ...

KEY WORDS: Time integration; nonlinear dynamics; energy conservation.

\section{INTRODUCTION}

Numerical time integration is used extensively to obtain the response of dynamic systems like flexible bodies, structures and electromechanical systems to dynamic loads. The preferred format is the single-step algorithm, in which the state-space generalized displacement and velocity vectors $\mathbf{u}$ and $\mathbf{v}$ are obtained at time $t_{n+1}$, when known at time $t_{n}$. The most common format is that of collocation, in which the equation of motion is satisfied at a sequence of points in time $\cdots, t_{n}, t_{n+1}$, and various forms of the Newmark scheme $[1,2,3,4]$ are probably the most used. In linear problems the algorithm can be characterized by a spectral analysis, which essentially is the free response of a single modal form, [5, 4]. An alternative,

*Correspondence to: Steen Krenk, Department of Mechanical Engineering, Building 403, Nils Koppel's Alle, DK-2800 Kongens Lyngby, Denmark. E-mail: sk@mek.dtu.dk

Copyright (C) 2014 John Wiley \& Sons, Ltd.

Prepared using nmeauth.cls [Version: 2010/05/13 v3.00] 
that gives less detailed information, but can be extended to non-linear systems, is the energy balance equation. An energy analysis of the linear Newmark algorithm reveals that with the exception of the simple 'average acceleration' form of the Newmark algorithm the energy as described by the algorithm does not correspond to to the mechanical energy of the original system, [6]. Thus, energy conservation in the computed response corresponds to conservation of the 'algorithmic energy', and thereby leads to fluctuations in the actual mechanical energy. In the case of non-linear systems also the 'average acceleration' scheme loses its energy balance properties. Attempts have been made to restore energy conservation by introducing it as an external constraint in the algorithm [7]. There have been further extensions to the introduction of momentum balance by an additional constraint [8] and combining the energy constraint with a high-frequency dissipation scheme [9], but a more detailed analysis [10] has demonstrated inconsistencies in the combination of the energy constraint and the dissipation scheme. In practice, undesirable energy fluctuations introduced by collocation algorithms are often reduced by introducing algorithmic damping, e.g. by the high-frequency damping procedures discussed in $[2,3,4]$, but clearly this is a sub-optimal solution to the lack of an accurate energy balance in nonlinear collocation methods.

Over the last two decades there has been a considerable effort in developing so-called energymomentum based nonlinear time integration algorithms. The basic idea is to integrate the equation - in state-space or Hamilton form - and then to represent the internal forces and the momentum defining relations in a format that leads to energy conservation. Thus, in contrast to the previous algorithms, the conservation properties are built into the algorithms, and due to the initial integration of the equations of motion the acceleration is not an independent variable of the formulation. Important early contributions to momentum-energy based algorithms were made by Simo and Wong [11] for rigid body motion and Simo and Tarnow [12] for linear elasticity in terms of the nonlinear quadratic Green strain, demonstrating that energy conservation can be obtained by evaluating the effective internal force at a suitably defined mean state within the integration interval. The formation of the internal force at an internal mean state has since become a standard procedure, and for continuum mechanics various choices of mean state have recently been discussed by Romero [13]. The mean state is formed at the particle level, and in the context of models built by finite elements this implies that the computation must revert to the element level to calculate the representative internal force at suitable Gauss points in this mean state. This breaks with the classic program structure of nonlinear finite element analysis, and may be a contributing factor to the rather slow spreading of conservative time integration procedures outside the academic community. For the special case of linear elasticity in terms of the nonlinear quadratic Green strain it was demonstrated in [14] that the mean state formulation at the element level can be replaced by introducing the increment of the geometric stiffness matrix, calculated at the global level. However, this property is linked specifically to linear elasticity with quadratic strains. An important extension of the energy conservation algorithms to more general nonlinear problems was made by Gonzalez [15, 16], who generalized the representation of the internal force to include an additive discrete energy gradient term, much in the vein of the gradient representation in optimization algorithms, [17]. An early scalar version of the secant style conservative integration was presented by Greenspan [18], and discrete gradient integrations methods have been developed for classic mechanics [19], non-linear elasticity [20], multibody dynamics [21], and mathematical physics [22]. 
In the energy-momentum approach the algorithms are typically formulated for an undamped system. A systematic algorithmic damping can be introduced by introducing a pair of suitably balanced displacement and velocity increment terms as described in $[23,14]$. While damping is typically necessary for convergence of nonlinear collocation methods, the algorithmic damping in energy-momentum based procedures are mainly useful for representing actual damping in the system and is unrelated to the convergence of the algorithm.

The present paper develops a general momentum-based time integration scheme with energy conservation. First the time-integrated equations are developed in state-space format, and the corresponding discrete energy balance equation is obtained, including a simple balanced algorithmic damping mechanism. Then an energy-conserving form of the internal force is derived for a system with quartic force potential. The idea is to parameterize the displacement within the time increment in terms of a scalar variable $\xi$ and then to develop the energy increment in terms of a modified Taylor expansion, in which the center-values of the statespace variables are replaced by their value at the interval end-points. It is demonstrated that energy conservation is obtained by adding a term containing the increment of the tangent stiffness matrix over the integration interval to the algebraic mean value of the internal force at the interval end-points. The new term containing the stiffness matrix increment is located at the same position in the algorithm as a viscous damping, and this explains the energy fluctuations experienced by collocation schemes for non-linear problems, where this term is missing. The tangent stiffness matrix is typically needed for iterative solution in implicit non-linear integration algorithms, and thus the introduction of the increment of the tangent stiffness matrix in the actual balance equation does not impose any additional computational requirements. An important special case of the quartic energy format is linear elastic systems with quadratic strains, often used in kinematically non-linear models and multi-body dynamics. Finally, the representation of the internal force is generalized to arbitrary energy potentials by a discrete gradient formulation based on the quartic representation in terms of interval end-point values. Some simple examples illustrate the use of the quartic format representation as well as its secant extension.

\section{BASIC EQUATIONS AND THE STATE SPACE FORMAT}

Let a dynamic system be described by a set of $n$ displacement components $\mathbf{u}=\left[u_{1}, \cdots, u_{n}\right]^{T}$. The system is assumed to have an internal energy $G(\mathbf{u})$. The forces are then given by the gradient of the internal energy in the form

$$
\mathbf{g}(\mathbf{u})=\nabla_{\mathbf{u}} G(\mathbf{u})
$$

where $\boldsymbol{\nabla}_{\mathbf{u}}$ denotes the derivatives with respect to the displacement components of $\mathbf{u}$. For convenience the resulting components are defined to be in column format. A simple standard form of the equations of motion is

$$
\mathbf{M} \ddot{\mathbf{u}}+\mathbf{C} \dot{\mathbf{u}}+\mathbf{g}(\mathbf{u})=\mathbf{f}(t),
$$

where the inertia is represented by the constant mass matrix $\mathbf{M}$, corresponding to models e.g. with concentrated masses or deformable solids represented in terms of isoparametric elements. A linear viscous damping has been introduced via the constant matrix $\mathbf{C}$. When introducing 
a discretized time integration scheme it may be advantageous to replace this representation of damping with alternative form, linked directly to the time integration algorithm - so-called algorithmic damping. The algorithmic damping representation is typically designed to provide increasing damping of high-frequency components, whereas direct representation of the viscous damping leaves the high-frequency components with diminishing damping, [5]. The external force is represented by the vector $\mathbf{f}=\left[f_{1}, \cdots, f_{n}\right]^{T}$, given either as a function of time or by a potential relation similar to $(1)$,

$$
\mathbf{f}(\mathbf{u})=\nabla_{\mathbf{u}} F(\mathbf{u})
$$

In the case of a force potential, the external force may be included directly by replacing the internal potential $G(\mathbf{u})$ by the combined potential $U(\mathbf{u})=G(\mathbf{u})-F(\mathbf{u})$. This procedure is straight forward and will not be discussed explicitly in the following derivations.

When dealing with time integration of dynamic systems it is convenient to use a statespace formulation in which the second order differential equation of motion (2) is replaced by two first order differential equations - a dynamic equation expressing a balance of the rate of change of the momentum with the external forces, and a kinematic equation defining the momentum vector. For systems with constant mass matrix like (2) it is convenient to represent the momentum as $\mathbf{M v}$ in terms of an independent velocity variable $\mathbf{v}=\dot{\mathbf{u}}$. The equations of motion can then be given in the symmetric state-space form

$$
\left[\begin{array}{cc}
\mathbf{C} & \mathbf{M} \\
\mathbf{M} & \mathbf{0}
\end{array}\right]\left[\begin{array}{c}
\dot{\mathbf{u}} \\
\dot{\mathbf{v}}
\end{array}\right]+\left[\begin{array}{c}
\mathbf{g}(\mathbf{u}) \\
-\mathbf{M v}
\end{array}\right]=\left[\begin{array}{c}
\mathbf{f}(t) \\
\mathbf{0}
\end{array}\right]
$$

There are two advantages of using the first order equations for the state-space vector $\left[\mathbf{u}^{T}, \mathbf{v}^{T}\right]$ : the definition of $\mathbf{v}(t)$ as being identical to the velocity $\dot{\mathbf{u}}(t)$ at all times can be relaxed and included in the approximate procedure, and full symmetry can be retained and related to energy conservation in a simple manner.

The energy balance equation is obtained directly from the state-space equations of motion (4) by pre-multiplication with $\left[\dot{\mathbf{u}}^{T},-\dot{\mathbf{v}}^{T}\right]$. The contributions from the two off-diagonal submatrices in the first term cancel, leaving the energy equation in the form

$$
\frac{d}{d t}\left[\frac{1}{2} \mathbf{v}^{T} \mathbf{M v}+G(\mathbf{u})\right]=\dot{\mathbf{u}}^{T} \mathbf{f}-\dot{\mathbf{u}}^{T} \mathbf{C} \dot{\mathbf{u}}
$$

In this relation it has been used that the rate of change of the internal energy $G(\mathbf{u})$ follows from its gradient by application of the 'chain rule' of differentiation as

$$
\frac{d}{d t} G(\mathbf{u})=\dot{\mathbf{u}}^{T} \nabla_{\mathbf{u}} G(\mathbf{u})=\dot{\mathbf{u}}^{T} \mathbf{g}(\mathbf{u}) .
$$

The key to energy conservation is to develop an algorithm that contains the equivalent relation for a finite time interval and thereby finite increments $\Delta G$ and $\Delta \mathbf{u}$. The equivalent gradient appearing in the discretized relation is often termed the 'finite derivative', and a key point of the present paper is to present a novel form of this finite derivative.

\subsection{Discrete state-space equations}

In single-step time integration algorithms the state-space variables $\mathbf{u}, \mathbf{v}$ are advanced from a time $t_{n}$ to the time $t_{n+1}=t_{n}+h$, a time increment $h$ later, by use of the system matrices and the history of the load vector within the time interval. Classic time integration methods, 
like the Newmark family, are typically based on matching the equations of motion at selected times. In contrast, momentum-based methods use a time-integrated form of the state-space equations (4) over the time interval $\left[t_{n}, t_{n+1}\right]$. The first term, containing the derivatives $\dot{\mathbf{u}}(t)$ and $\dot{\mathbf{v}}(t)$, can be integrated exactly, giving the finite increments $\Delta \mathbf{u}$ and $\Delta \mathbf{v}$ over the time interval,

$$
\left[\begin{array}{cc}
\mathbf{C} & \mathbf{M} \\
\mathbf{M} & \mathbf{0}
\end{array}\right]\left[\begin{array}{c}
\Delta \mathbf{u} \\
\Delta \mathbf{v}
\end{array}\right]+\left[\begin{array}{c}
\int \mathbf{g}(\mathbf{u}) d t \\
-\int \mathbf{M} \mathbf{v} d t
\end{array}\right]=\left[\begin{array}{c}
\int \mathbf{f} d t \\
\mathbf{0}
\end{array}\right] .
$$

Recalling that contributions to the external force that are expressed in terms of a potential will be included in the potential function $G(\mathbf{u})$, the time integral of the remaining external forces is represented as the interval length $h$ times the arithmetic mean of the end-point values $\overline{\mathbf{f}}=\frac{1}{2}\left(\mathbf{f}_{n}+\mathbf{f}_{n+1}\right)$, and the velocity integral is similarly represented by its mean value $\overline{\mathbf{v}}=\frac{1}{2}\left(\mathbf{v}_{n}+\mathbf{v}_{n+1}\right)$.

The potential force $\boldsymbol{\nabla}_{\mathbf{u}} G(\mathbf{u})$ is generally non-linear in $\mathbf{u}$ and should therefore not be represented by the arithmetic mean of its value at the end-points of the integration interval. The integral of the potential is expressed in terms of a representative value of the internal force $\mathbf{g}_{*}$ in the form

$$
\int_{n}^{n+1} \nabla_{\mathbf{u}} G(\mathbf{u}) d t \simeq h \nabla_{\mathbf{u}} G_{*}=h \mathbf{g}_{*} .
$$

The representative 'mean' value of the internal force vector $\mathbf{g}_{*}=\nabla_{\mathbf{u}} G_{*}$ is determined as a finite gradient, satisfying the energy increment condition

$$
\Delta G=\Delta \mathbf{u}^{T} \nabla_{\mathbf{u}} G_{*}=\Delta \mathbf{u}^{T} \mathbf{g}_{*} .
$$

In terms of this notation the discretized equations of motion take the form

$$
\left[\begin{array}{cc}
\mathbf{C} & \mathbf{M} \\
\mathbf{M} & \mathbf{0}
\end{array}\right]\left[\begin{array}{c}
\Delta \mathbf{u} \\
\Delta \mathbf{v}
\end{array}\right]+h\left[\begin{array}{c}
\mathbf{g}_{*} \\
-\mathbf{M} \overline{\mathbf{v}}
\end{array}\right]=h\left[\begin{array}{c}
\overline{\mathbf{f}} \\
\mathbf{0}
\end{array}\right] .
$$

It is observed that in this format the viscous damping term is represented by $\mathbf{C} \Delta \mathbf{u}$ instead of $\mathbf{C} h \overline{\mathbf{v}}$ which is the typical form in collocation algorithms like the Newmark family $[1,2,3]$. The present formulation places viscous damping in the upper block diagonal of the first term, a location that is more consistent and robust than the location in the upper right corner of the second block matrix corresponding to Newmark type algorithms, [6].

\subsection{Energy balance equation}

The energy relation for the discrete algorithm corresponding to (9) follows by premultiplication with $\left[\Delta \mathbf{u}^{T},-\Delta \mathbf{v}^{T}\right]$,

$$
\left[\Delta \mathbf{u}^{T},-\Delta \mathbf{v}^{T}\right]\left(\left[\begin{array}{cc}
\mathbf{C} & \mathbf{M} \\
\mathbf{M} & \mathbf{0}
\end{array}\right]\left[\begin{array}{c}
\Delta \mathbf{u} \\
\Delta \mathbf{v}
\end{array}\right]+h\left[\begin{array}{c}
\mathbf{g}_{*} \\
-\mathbf{M} \overline{\mathbf{v}}
\end{array}\right]\right)=h \Delta \mathbf{u}^{T} \overline{\mathbf{f}} .
$$

In the first matrix the contributions from the off-diagonal sub-matrices cancel, while the diagonal term represents the dissipation by viscous damping. The $\mathbf{v}$-contributions from the second matrix are rewritten by use of the relation

$$
\left(\mathbf{v}_{n+1}^{T}-\mathbf{v}_{n}^{T}\right) \mathbf{M}\left(\mathbf{v}_{n+1}+\mathbf{v}_{n}\right)=\left[\mathbf{v}^{T} \mathbf{M} \mathbf{v}\right]_{n}^{n+1},
$$


following from symmetry of the mass matrix M. After division by the time increment $h$ and use of the relation (10) the energy equation takes the form

$$
\left[\frac{1}{2} \mathbf{v}^{T} \mathbf{M} \mathbf{v}+G(\mathbf{u})\right]_{n}^{n+1}=\Delta \mathbf{u}^{T} \overline{\mathbf{f}}-\frac{1}{h} \Delta \mathbf{u}^{T} \mathbf{C} \Delta \mathbf{u} .
$$

This is recognized as the finite increment form of the energy balance equation (5).

\subsection{Consistent algorithmic damping}

In the discrete energy balance equation (13) the viscous dissipation is represented via a quadratic expression in the displacement increment $\Delta \mathbf{u}$. The discretization leads to diminishing damping for the high frequencies beyond the aliasing limit. This problem can be remedied by introducing a dissipation that combines quadratic terms in $\Delta \mathbf{u}$ and $\Delta \mathbf{v},[23,24]$. The corresponding algorithm contains balanced diagonal terms in the block diagonal of the first matrix,

$$
\left[\begin{array}{cc}
\mathbf{C}+\frac{1}{2} \alpha h \mathbf{K}_{\alpha} & \mathbf{M} \\
\mathbf{M} & -\frac{1}{2} \alpha h \mathbf{M}
\end{array}\right]\left[\begin{array}{c}
\Delta \mathbf{u} \\
\Delta \mathbf{v}
\end{array}\right]+h\left[\begin{array}{c}
\mathbf{g}_{*} \\
-\mathbf{M} \overline{\mathbf{v}}
\end{array}\right]=h\left[\begin{array}{l}
\overline{\mathbf{f}} \\
\mathbf{0}
\end{array}\right] .
$$

In this formula $\mathbf{K}_{\alpha}$ is a representative value of the stiffness matrix, discussed further in Section 4 , and $\alpha$ is a non-dimensional parameter controlling the magnitude of the algorithmic damping. The corresponding energy balance equation is found as above,

$$
\left[\frac{1}{2} \mathbf{v}^{T} \mathbf{M} \mathbf{v}+G(\mathbf{u})\right]_{n}^{n+1}=\Delta \mathbf{u}^{T} \overline{\mathbf{f}}-\frac{1}{h} \Delta \mathbf{u}^{T} \mathbf{C} \Delta \mathbf{u}-\frac{1}{2} \alpha\left[\Delta \mathbf{v}^{T} \mathbf{M} \Delta \mathbf{v}+\Delta \mathbf{u}^{T} \mathbf{K}_{\alpha} \Delta \mathbf{u}\right] .
$$

The algorithmic damping is seen to consist of two quadratic terms in $\Delta \mathbf{v}$ and $\Delta \mathbf{u}$, respectively, balanced as in a mechanical energy with stiffness $\mathbf{K}_{\alpha}$.

For linear systems there is a simple low-frequency relation between the algorithmic damping parameter $\alpha$ and the algorithmic damping ratio $\zeta_{a}$ of a particular mode with natural angular frequency $\omega$. Consider the free response of a system without viscous damping. In the lowfrequency regime the displacement and velocity increments on the right side of the energy balance can be expressed approximately by the corresponding time derivatives. For a lightly damped harmonic response with angular frequency $\omega$ this leads to the following approximate form of the energy equation

$$
\Delta E \simeq \alpha(\omega h)^{2} E,
$$

For a linear oscillator the similar result is

$$
\frac{d E}{d t} \simeq 2 \zeta_{a} \omega E
$$

from which a low-frequency approximation for the algorithm damping ratio then follows as

$$
\zeta_{a} \simeq \frac{1}{2} \alpha \omega h .
$$

In the low-frequency region the algorithmic damping ratio is proportional to the angular frequency, corresponding closely to the stiffness component in Rayleigh damping.

\section{CONSISTENT INTERNAL FORCE}

In collocation methods the effective internal force $\mathbf{g}_{*}$ appearing in the discretized equations (10) typically has the form of a weighted average of the internal force at the interval end points 
$t_{n}$ and $t_{n+1}$. For nonlinear problems this format is unable to represent the energy conservation condition (9). In this section an energy conserving form is obtained in two steps. First, an exact conservative formulation is found for systems with internal energy as a quartic function of the displacements. For these systems the effective internal force is expressed directly in terms of the mean internal force $\bar{g}$ and the increment of the tangent stiffness $\Delta \mathbf{K}$. Subsequently, a general secant formulation is derived, based on the quartic representation and the increment of the internal energy $\Delta G$.

\subsection{Equivalent internal force for quartic potential}

In the case of a quartic energy function the representation of $\mathbf{g}_{*}$ is obtained from a suitably modified form of the Taylor expansion of the potential $G(\mathbf{u})$. For this purpose the displacement increment is parameterized over the time interval $\left[t_{n}, t_{n+1}\right]$ as

$$
\mathbf{u}=\overline{\mathbf{u}}+\xi \Delta \mathbf{u}, \quad-\frac{1}{2} \leq \xi \leq \frac{1}{2} .
$$

This gives the following expression for increment of the internal energy,

$$
\Delta G=\int_{n}^{n+1} \mathbf{g}(\mathbf{u})^{T} d \mathbf{u}=\Delta \mathbf{u}^{T} \int_{-1 / 2}^{1 / 2} \mathbf{g}(\overline{\mathbf{u}}+\xi \Delta \mathbf{u}) d \xi .
$$

The internal force is now expanded in terms of the scalar parameter $\xi$ as

$$
\mathbf{g}(\mathbf{u})=\mathbf{g}(\overline{\mathbf{u}})+\xi \mathbf{g}^{\prime}(\overline{\mathbf{u}})+\frac{1}{2} \xi^{2} \mathbf{g}^{\prime \prime}(\overline{\mathbf{u}})+\cdots
$$

When substituting this expansion into the integral in (20) it is seen that only the even powers of $\xi$ contribute, and when including only the first two contributing terms

$$
\Delta G=\Delta \mathbf{u}^{T}\left[\mathbf{g}(\overline{\mathbf{u}})+\frac{1}{24} \mathbf{g}^{\prime \prime}(\overline{\mathbf{u}})+\mathrm{O}\left(h^{4}\right)\right],
$$

where it has been used that $\Delta \mathbf{u}$ is of order $h$, and that each derivative with respect to $\xi$ introduces a factor $\Delta \mathbf{u}$ of order $h$. For a quartic potential $\mathrm{g}^{\mathrm{iv}}$ and higher order derivatives vanish identically, and in this case the first two terms constitute an exact representation.

The terms inside the square brackets define the effective internal force as

$$
\mathbf{g}_{*}=\mathbf{g}(\overline{\mathbf{u}})+\frac{1}{24} \mathbf{g}^{\prime \prime}(\overline{\mathbf{u}})+\mathrm{O}\left(h^{4}\right) .
$$

The first term in this representation is evaluated in the mean displacement state $\overline{\mathbf{u}}$. This would be a serious computational drawback, and the representation is therefore reformulated by use of the Taylor expansion (21) to express the algebraic mean of the internal force,

$$
\frac{1}{2}\left[\mathbf{g}_{n+1}+\mathbf{g}_{n}\right]=\mathbf{g}(\overline{\mathbf{u}})+\frac{1}{8} \mathbf{g}^{\prime \prime}(\overline{\mathbf{u}})+\mathrm{O}\left(h^{4}\right) .
$$

When this relation is used to eliminate $\mathbf{g}(\overline{\mathbf{u}})$ in $(23)$ the effective force is obtained in the form

$$
\mathbf{g}_{*}=\frac{1}{2}\left[\mathbf{g}_{n+1}+\mathbf{g}_{n}\right]-\frac{1}{12} \mathbf{g}^{\prime \prime}(\overline{\mathbf{u}})+\mathrm{O}\left(h^{4}\right) .
$$

It now remains to obtain a representation of the second term without explicit reference to the mean state $\overline{\mathbf{u}}$.

The second term is expressed in terms of the increment of the tangent stiffness, $\Delta \mathbf{K}$. In order to obtain this expression the first derivative of the internal force with respect to the 
non-dimensional parameter $\xi$ is expressed as

$$
\mathbf{g}^{\prime}(\mathbf{u})=\frac{d \mathbf{g}}{d \mathbf{u}} \Delta \mathbf{u}=\mathbf{K}(\mathbf{u}) \Delta \mathbf{u} .
$$

In the present formulation the displacement increment $\Delta \mathbf{u}$ is fixed, and the second derivative of the internal force with respect to the non-dimensional parameter $\xi$ therefore follows by a secant representation as

$$
\mathbf{g}^{\prime \prime}(\mathbf{u})=\frac{d \mathbf{K}}{d \xi} \Delta \mathbf{u} \simeq \frac{\Delta \mathbf{K}}{\Delta \xi} \Delta \mathbf{u}=\Delta \mathbf{K} \Delta \mathbf{u} .
$$

Substitution of this representation into the expansion (25) then gives the final form

$$
\mathbf{g}_{*}=\frac{1}{2}\left[\mathbf{g}_{n+1}+\mathbf{g}_{n}\right]-\frac{1}{12} \Delta \mathbf{K} \Delta \mathbf{u}+\mathrm{O}\left(h^{4}\right) .
$$

In this form the effective internal force is expressed to fourth order entirely by the internal force and the stiffness matrix at the integration interval end-points.

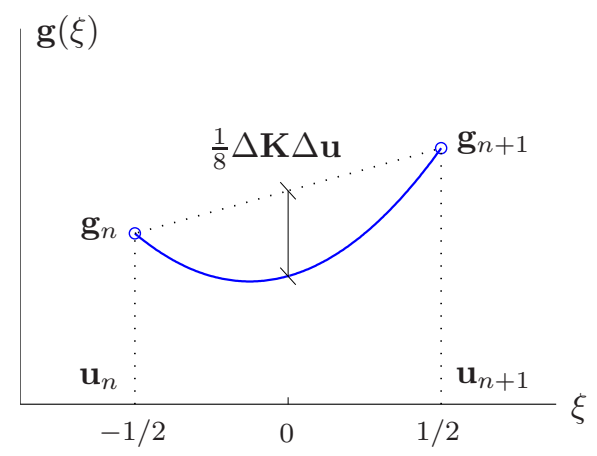

Figure 1. Integration of parabolic representation in terms of $\xi$.

The result (28) for the effective internal force $\mathbf{g}_{*}$ is illustrated in Fig. 1. The figure shows the development of the internal force $\mathbf{g}(\xi)$ in terms of the formal interpolation parameter $\xi$. The slope of the curve $d \mathbf{g} / d \xi$ is given by (26) at the interval end-points $\mathbf{u}_{n}$ and $\mathbf{u}_{n+1}$ as $\mathbf{K}_{n} \Delta \mathbf{u}$ and $\mathbf{K}_{n+1} \Delta \mathbf{u}$, respectively. Only the symmetric part, described by the difference of these derivatives, contributes to the integral expression (20) for the increment of the internal energy $\Delta G$. This contribution may be represented as a parabola with inclination $\pm \frac{1}{2} \Delta \mathbf{K} \Delta \mathbf{u}$ at $\xi= \pm \frac{1}{2}$. The height of the parabola at the center $\xi=0$ equals half of the slope times the distance $\frac{1}{2}$ from the center to the interval ends. This gives the parabola height $\frac{1}{8} \Delta \mathbf{K} \Delta \mathbf{u}$ as shown in the figure. Integration over the $\xi$-interval of length 1 then gives the effective force $\mathbf{g}_{*}$ as expressed by the two first terms of (28).

\subsection{Extension to general internal potential}

It is desirable if the effective internal force $\mathbf{g}_{*}$ satisfies the energy increment condition (9). For systems with a quartic internal energy function $G(\mathbf{u})$ this is attained when using the two-term representation (28). For a more general form of the internal energy function the energy conservation property can be satisfied by introducing a higher order modification of the internal force such that the finite energy increment condition (9) is satisfied. This procedure, introduced by Gonzalez [16], is typically based on an interior point evaluation of the internal 
force, $[16,20,13]$. In the present formulation the secant form of the internal force is obtained as an extension of the explicit global end-point representation (28) for a quartic potential function,

$$
\mathbf{g}_{\mathrm{q}}=\frac{1}{2}\left[\mathbf{g}_{n+1}+\mathbf{g}_{n}\right]-\frac{1}{12} \Delta \mathbf{K} \Delta \mathbf{u} .
$$

The secant form is obtained by addition of a higher-order term that modifies the energy increment associated with the actual displacement increment $\Delta \mathbf{u}$. In the present context it is convenient to use the formulation

$$
\mathbf{g}_{*}=\mathbf{g}_{\mathrm{q}}+\frac{\left(G_{n+1}-G_{n}\right)-\Delta \mathbf{u}^{T} \mathbf{g}_{\mathrm{q}}}{\Delta \mathbf{u}^{T} \Delta \mathbf{g}} \Delta \mathbf{g}
$$

in terms of the increment of the internal force $\Delta \mathbf{g}$ over the current time increment. It follows immediately by pre-multiplication with $\Delta \mathbf{u}$ that the algorithmic internal force $\mathbf{g}_{*}$ defined by the secant form (30) satisfies the finite energy increment relation

$$
\Delta \mathbf{u}^{T} \mathbf{g}_{*}=G_{n+1}-G_{n},
$$

as required for energy conservation. If, in any time increment the denominator $\Delta \mathbf{u}^{T} \Delta \mathbf{g}$ is 'small', the increment of the potential $G$ is small, and the last term containing the potentially ill-conditioned fraction is simply omitted. The energy balance is re-established over the next time increment. This is a common feature of using a secant correction term.

In the formula (30)the numerator of the correction term is the remaining part of the potential increment $\Delta G$, when the contribution from a quartic expansion of the energy $G$ has been subtracted, and thus

$$
\left(G_{n+1}-G_{n}\right)-\Delta \mathbf{u}^{T} \mathbf{g}_{\mathrm{q}}=\mathrm{O}\left(h^{5}\right) .
$$

It is a characteristic feature of the present formulation that the numerator of the secant correction is of order $\mathrm{O}\left(h^{5}\right)$, and thus the correction term vanishes for a quartic potential function, leaving $\mathbf{g}_{*}=\mathbf{g}_{\mathrm{q}}$.

\subsection{Momentum balance}

The present formulation is momentum based in the sense that the dynamic differential equations are integrated over time to produce a generalized momentum balance equation, represented by the first of the equations in (14). However, the problem setting as well as the solution method does not rely on any special form of the equations, like that associated with dynamic motion of flexible bodies, and can include e.g. electromechanical effects present in transducers and actuators. When rearranging the terms this equation becomes an expression for the generalized momentum increment over the time integration increment $\left[t_{n}, t_{n+1}\right]$,

$$
\mathbf{M} \Delta \mathbf{v}=h \overline{\mathbf{f}}-h \mathbf{g}_{*}-\frac{1}{2} \alpha h \mathbf{K}_{\alpha} \Delta \mathbf{u}-\mathbf{C} \Delta \mathbf{u} .
$$

Within a finite element context the vector on the right is the collection of contributions to the generalized momentum vector from each of the nodes, and in simple mechanical models the connection to the momentum follows directly. In a mechanical system without supports neither the internal force $\mathbf{g}$ nor the stiffness matrix $\mathbf{K}$ can produce a resulting force. The change in the total momentum is obtained by summation of the nodal contributions, and as the algorithmic force $\mathbf{g}_{*}$ in the present formulation is formed by combinations of internal forces and stiffness matrices, these will not contribute. Neither will the term $\mathbf{K}_{\alpha} \Delta \mathbf{u}$, which is either formed from 
stiffness matrices, or from the increment of the internal force $\Delta \mathrm{g}$ as explained in the following section together with the specifics of the algorithm. The last term $\mathbf{C} \Delta \mathbf{u}$ represents the effect of the viscous damping in the model. If using the Rayleigh damping assumption the damping matrix $\mathbf{C}$ is a linear combination of the mass matrix $\mathbf{M}$ and the stiffness matrix $\mathbf{K}$. The stiffness contribution is formed from instantaneous stiffness matrices, e.g. in the form of a mean value, and thus does not contribute to the resulting generalized momentum increment. Contrary with a mass matrix contribution, which will reduce the momentum, because this term implicitly makes reference to absolute motion. In the absence of any reference to absolute motion in the damping matrix $\mathbf{C}$ the increment of the total momentum is determined by the impulse of the external forces, represented by a contracted form of $h \overline{\mathbf{f}}$. Thus, momentum balance would occur, if $h \overline{\mathbf{f}}$ represents the exact external force time integral. In the case of structures with supports the general features are the same, but the detailed time distribution of the reaction forces is subject to approximate representation.

\section{TIME INTEGRATION ALGORITHM}

The full state-space format $\mathbf{u}, \mathbf{v}$ was introduced in (10) and (14) to identify the structure of the algorithm. In the actual computation it is advantageous to eliminate the explicit dependence on the velocity components $\mathbf{v}_{n+1}$ in the matrix equations. The formulation takes a particularly simple form if the algorithmic damping parameter $\alpha$ is absorbed into a dissipation parameter

$$
\kappa=1+\alpha .
$$

In the second of the state-space equations (14) the mass matrix $\mathbf{M}$ is a common factor that can be omitted, leaving the following relation between velocity and displacement components,

$$
\kappa \Delta \mathbf{v}=\frac{2}{h} \Delta \mathbf{u}-2 \mathbf{v}_{n}
$$

This relation is now used to eliminate the velocity increment $\Delta \mathbf{v}$ from the first state-space equation in (14), which then takes the form

$$
\kappa\left[\left(\frac{2}{\kappa h}\right)^{2} \mathbf{M}+\frac{2}{\kappa h} \mathbf{C}\right] \Delta \mathbf{u}+\left(2 \mathbf{g}_{*}+\alpha \mathbf{K}_{\alpha} \Delta \mathbf{u}\right)=\mathbf{f}_{n+1}+\mathbf{f}_{n}+\frac{4}{\kappa h} \mathbf{M v}_{n} .
$$

This is a non-linear equation in $\mathbf{u}_{n+1}$. It is reformulated slightly before introducing it into the solution algorithm.

The concept of algorithmic damping is primarily useful in connection with oscillatory motion, which assumes that the local stiffness matrix $\mathbf{K}$ is positive definite. The contribution from the damping stiffness matrix $\mathbf{K}_{\alpha}$ is then conveniently defined by the secant relation

$$
\mathbf{K}_{\alpha} \Delta \mathbf{u}=\Delta \mathbf{g}
$$

With this representation of the algorithmic damping and use of the secant form (30) for the effective internal force, the internal forces in the last parenthesis on the left side of the equality in (36) take the form

$$
2 \mathbf{g}_{*}+\alpha \Delta \mathbf{g}=\kappa \Delta \mathbf{g}+2 \mathbf{g}_{n}-\frac{1}{6} \Delta \mathbf{K} \Delta \mathbf{u}+2 \eta \Delta \mathbf{g} .
$$


where for convenience the non-dimensional scalar factor of the secant approximation term in (30) is denoted

$$
\eta=\frac{\Delta G-\Delta \mathbf{u}^{T} \mathbf{g}_{\mathrm{q}}}{\Delta \mathbf{u}^{T} \Delta \mathbf{g}}
$$

The coefficient $\eta$ depends on the displacement states $\mathbf{u}_{n}$ and $\mathbf{u}_{n+1}$ at the beginning and the end

of the current integration interval and is of order $\eta=\mathrm{O}\left(\|\Delta \mathbf{u}\|^{3}\right)$. In the formula (38) the two last terms are the effect of non-linearity corresponding to a quartic internal energy potential $G(\mathbf{u})$ and an additional secant term for exact energy balance for a general energy potential. It is observed that both these terms have a format, that couples the states at $t_{n}$ and $t_{n+1}$, and thus these terms can not be accommodated within the format of a collocation based time integration method.

When the representation (38) for the internal force term is introduced, the first state space equation (36) takes the somewhat more structured form

$$
\kappa\left[\left(\frac{2}{\kappa h}\right)^{2} \mathbf{M}+\frac{2}{\kappa h} \mathbf{C}\right] \Delta \mathbf{u}-\frac{1}{6} \Delta \mathbf{K} \Delta \mathbf{u}+(\kappa+2 \eta) \Delta \mathbf{g}=\mathbf{f}_{n+1}+\mathbf{f}_{n}-2 \mathbf{g}_{n}+\frac{4}{\kappa h} \mathbf{M} \mathbf{v}_{n} .
$$

Advancing the solution one time step involves iterative solution of the non-linear equation of motion (40) for $\Delta \mathbf{u}$, followed by evaluation of the velocity increment $\Delta \mathbf{v}$ by use of the linear relation (35).

\subsection{The iteration process}

In the solution process the equation (40) for $\mathbf{u}_{n+1}$ is written in the form of a residual, formed by the difference between the terms on the right side and the terms on the left side,

$$
\mathbf{r}=\mathbf{f}_{n+1}+\mathbf{f}_{n}-2 \mathbf{g}_{n}-(\kappa+2 \eta) \Delta \mathbf{g}+\frac{1}{6} \Delta \mathbf{K} \Delta \mathbf{u}-\kappa\left[\left(\frac{2}{\kappa h}\right)^{2} \mathbf{M}+\frac{2}{\kappa h} \mathbf{C}\right] \Delta \mathbf{u}+\frac{4}{\kappa h} \mathbf{M v}_{n} .
$$

Iteration by the Newton-Raphson procedure essentially amounts to calculating the value of the residual $\mathbf{r}$, and if this is not sufficiently close to zero, a linearized increment $\delta \mathbf{r}$ is calculated in order to make the residual vanish, i.e.

$$
\mathbf{r}+\delta \mathbf{r}=\mathbf{r}+\frac{\partial \mathbf{r}}{\partial \mathbf{u}} \delta \mathbf{u}=\mathbf{0}
$$

The last equality is written in the finite increment form

$$
\mathbf{K}_{*} \delta \mathbf{u}=\mathbf{r} .
$$

with the stiffness matrix of the residual force defined as $\mathbf{K}_{*}=-\partial \mathbf{r} / \partial \mathbf{u}$.

A full differentiation of the residual force (41) would introduce higher derivatives and fairly complicated expressions due to the presence of the stiffness matrix increment $\Delta \mathbf{K}$ and the secant approximation factor $\eta\left(\mathbf{u}_{n}, \mathbf{u}_{n+1}\right)$. Furthermore, the details of these expressions would depend on the specific structure of the energy potential $G(\mathbf{u})$ and the corresponding internal force $\mathbf{g}(\mathbf{u})$. However, a simple and explicit approximate result is available for the part of the problem corresponding to a local quartic energy function representation. This result is obtained by representing the algorithmic force $\mathbf{g}_{*}$ as an integral of the parameterized increment $\xi \Delta \mathbf{u}$ illustrated in Fig. 1. Let the algorithmic internal force be evaluated by integration over $\xi$ using Simpson's rule,

$$
\mathbf{g}_{*} \simeq \frac{1}{6}\left(\mathbf{g}_{n+1}+\mathbf{g}_{n}\right)+\frac{2}{3} \mathbf{g}(\overline{\mathbf{u}})
$$


Table I. Energy consistent algorithm with dissipation.

1) Initial conditions:

$\mathbf{u}_{0}, \mathbf{v}_{0}$

2) Prediction step:

$\Delta \mathbf{u}=h \mathbf{v}_{n}$

3) Residual calculation:

$\mathbf{u}_{n+1}=\Delta \mathbf{u}+\mathbf{u}_{n}$

If $G(\mathbf{u})$ higher order than quartic and $\left|\Delta \mathbf{u}^{T} \Delta \mathbf{g}\right|>\varepsilon_{\mathbf{g}}$

$\eta=\left[\Delta G-\Delta \mathbf{u}^{T} \overline{\mathbf{g}}+\frac{1}{12} \Delta \mathbf{u}^{T} \Delta \mathbf{K} \Delta \mathbf{u}\right] /\left(\Delta \mathbf{u}^{T} \Delta \mathbf{g}\right)$ else $\eta=0$

$\mathbf{K}_{\mathrm{d}}=(2 / \kappa h)^{2} \mathbf{M}+(2 / \kappa h) \mathbf{C}$

$\mathbf{r}=\mathbf{f}_{n+1}+\mathbf{f}_{n}-2 \mathbf{g}_{n}-(\kappa+2 \eta) \Delta \mathbf{g}-\left(\kappa \mathbf{K}_{\mathrm{d}}-\frac{1}{6} \Delta \mathbf{K}\right) \Delta \mathbf{u}+(4 / \kappa h) \mathbf{M} \mathbf{v}_{n}$

4) Displacement sub-increment:

$$
\begin{aligned}
& \mathbf{K}_{*}=\kappa\left[\mathbf{K}+\mathbf{K}_{\mathrm{d}}\right]-\frac{1}{3} \Delta \mathbf{K} \\
& \delta \mathbf{u}=\mathbf{K}_{*}^{-1} \mathbf{r} \\
& \Delta \mathbf{u}=\Delta \mathbf{u}+\delta \mathbf{u}
\end{aligned}
$$

If $\|\mathbf{r}\|>\varepsilon_{\mathbf{r}}$ or $\|\delta \mathbf{u}\|>\varepsilon_{\mathbf{u}}$ repeat from 3 ).

5) State vector update:

$$
\begin{aligned}
& \mathbf{u}_{n+1}=\mathbf{u}_{n}+\Delta \mathbf{u} \\
& \mathbf{v}_{n+1}=\mathbf{v}_{n}+(2 / \kappa h)\left(\Delta \mathbf{u}-h \mathbf{v}_{n}\right)
\end{aligned}
$$

6) Return to 2) for new time step, or stop.

The corresponding differential increment of $2 \mathbf{g}_{*}$ to be used in the increment of the residual force then is

$$
\delta\left(2 \mathbf{g}_{*}\right) \simeq \frac{1}{3} \mathbf{K}_{n+1} \delta \mathbf{u}+\frac{4}{3} \mathbf{K}(\overline{\mathbf{u}})\left(\frac{1}{2} \delta \mathbf{u}\right)=\mathbf{K}_{n+1} \delta \mathbf{u}-\frac{2}{3}\left(\mathbf{K}_{n+1}-\mathbf{K}(\overline{\mathbf{u}})\right) \delta \mathbf{u} .
$$

In this formula the last difference in principle requires the evaluation of the stiffness matrix at the mid-point $\overline{\mathbf{u}}$. However, within the quartic potential assumption the contribution to the algorithmic force $\mathbf{g}_{*}$ depends on the end-point values and the second derivative within the interval, represented by the increment of $\mathbf{K}$. Therefore, assuming that $\mathbf{K}_{n+1}-\mathbf{K}(\overline{\mathbf{u}}) \simeq \frac{1}{2} \Delta \mathbf{K}$, the differential increment $\delta\left(2 \mathbf{g}_{*}\right)$ takes the approximate form

$$
\delta\left(2 \mathbf{g}_{*}\right) \simeq\left(\mathbf{K}_{n+1}-\frac{1}{3} \Delta \mathbf{K}\right) \delta \mathbf{u}
$$

This implies that $\delta(\Delta \mathbf{K} \Delta \mathbf{u}) \simeq 2 \Delta \mathbf{K} \delta \mathbf{u}$ in the present context of a contribution to an integral mean value. When using this representation of the contribution from the internal force the stiffness matrix of the residual force takes the simple form

$$
\mathbf{K}_{*}=-\frac{\partial \mathbf{r}}{\partial \mathbf{u}} \simeq \kappa\left[\left(\frac{2}{\kappa h}\right)^{2} \mathbf{M}+\frac{2}{\kappa h} \mathbf{C}+\mathbf{K}\right]-\frac{1}{3} \Delta \mathbf{K} .
$$


The terms in the parenthesis correspond to the coefficient matrix of a similar linear problem, while the last term represents the effect of the coupling over the integration interval present in non-linear problems.

\subsection{The algorithm}

The implementation of the algorithm is illustrated in pseudo code in Table I with $\mathbf{K}_{\mathrm{d}}$ denoting the dynamic part of the algorithmic stiffness matrix. The algorithm deals specifically with the energy balance over the interval $\left[t_{n}, t_{n+1}\right]$, and the equation of motion is therefore not matched at specific points, but includes the extra term $\Delta \mathbf{K} \Delta \mathbf{u}$ that combines the equations of motion at $t_{n}$ and $t_{n+1}$. The method is formulated entirely in the state-space variables $\mathbf{u}$ and $\mathbf{v}$, and thus the acceleration is not evaluated explicitly in the algorithm. The absence of an explicit value of the acceleration leads to the use of a simple predictor. If needed, the acceleration can be evaluated from the equation of motion at the corresponding time or by a suitable interpolation formula. The algorithmic damping is accounted for by choosing the coefficient $\kappa$ slightly larger than unity. This leads to a forward weighting of the internal force and a reduction of damping and inertial terms via an equivalent time step length $\kappa h$.

\section{EXAMPLES}

The properties of the energy-momentum algorithm developed above are illustrated by simple dynamic systems in the following examples. The first example illustrates the application of the algorithm on the Duffing oscillator, where the energy function is a quartic function of the displacement, and the simple form without secant correction therefore exhibits energy conservation. In the second example the problem is generalized to an oscillator with non-linear stiffness represented by a sinh-function. In this case energy conservation is obtained via the secant correction in the algorithm. In both cases an expression of the period of oscillation is available in terms of the complete elliptic integral of the first kind, and the second order convergence of the algorithm is demonstrated by comparison with these results. The last example illustrates energy conservation and algorithmic dissipation for an elastic pendulum with a slow swinging mode and a fast axial extension mode described in terms of Green strain.

\subsection{Duffing oscillator}

The Duffing oscillator is often used to model the dynamics of a single-degree-of-freedom system with a simple non-linear stiffness represented as a combination of a linear and a cubic term. It is represented by the equation of motion for the mass $m$ as

$$
m \frac{d^{2} u}{d t^{2}}+g(u)=f(t)
$$

with internal force $g(u)$ and internal energy $G(u)$ given in terms of the initial stiffness $k$ and the stiffness non-linearity parameter $\lambda$ as

$$
g(u)=k u\left(1+\lambda^{2} u^{2}\right), \quad G(u)=\frac{1}{2} k u^{2}\left(1+\frac{1}{2} \lambda^{2} u^{2}\right) .
$$

The tangent stiffness follows from differentiation of the internal force $g(x)$ as

$$
K(u)=k\left(1+3 \lambda^{2} u^{2}\right)
$$



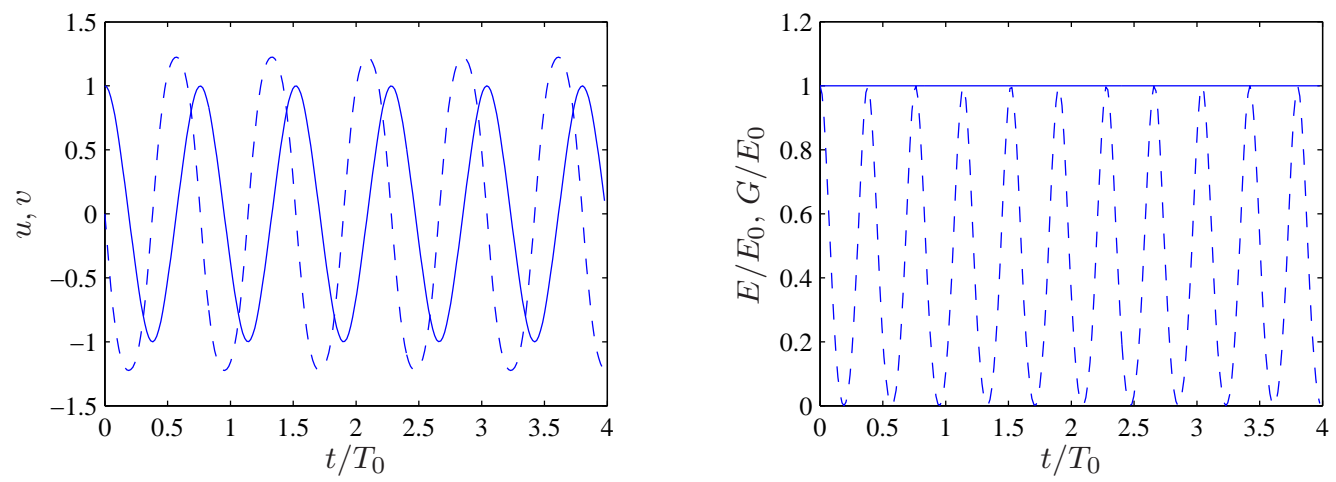

Figure 2. Duffing oscillator $h / T_{0}=0.08$. a) Response $u(t)-, v(t) / \omega_{0}--$, b) Energy $E(t)-, G(t)-$.

The energy potential $G(u)$ is of degree four in the displacement $u$, and it is easily verified that the secant correction vanishes.

Figure 2 illustrates the response of a Duffing oscillator with $m=1.0, k=1.0, \lambda=1.0$, and a time step of $h=0.01$. These parameters correspond to a reference period of $T_{0}=2 \pi$. Time histories of $u, v$ for initial conditions $u_{0}=1.0, v_{0}=0.0$ are shown in Fig. 2a. The initial displacement $u_{0}$ is the maximum displacement, and it follows from (50) that the stiffness varies between 1.0 and 4.0 during an oscillation, indicating a fairly non-linear oscillation. This is illustrated by the fact that the normalized velocity $v(t) / \omega_{0}$ reaches a higher maximum value than the displacement $u(t)$. Figure $2 \mathrm{~b}$ shows the total energy $E(t)$ and the elastic energy $G(u)$. While the total energy is conserved within about 12 digits, the potential energy shows regular fluctuations between the maximum level $E_{0}$ and zero.

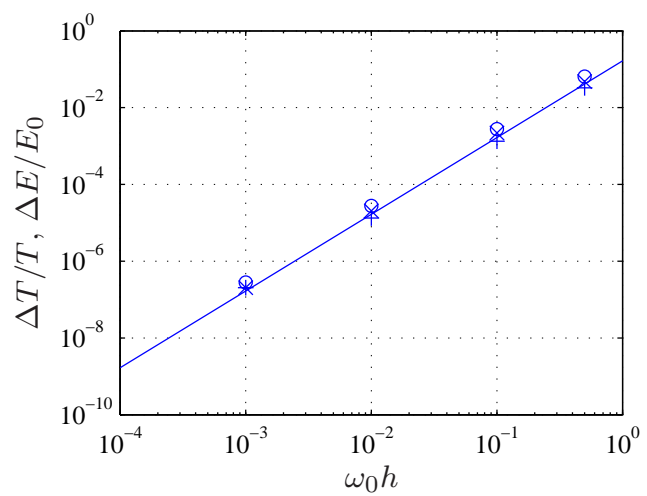

Figure 3. Duffing oscillator $\lambda u_{m}=1.0$ : Conservative $\Delta T / T: \times$, Newmark $\Delta T / T:+, \Delta E / E_{0}: \circ$.

The free-vibration period $T$ of the Duffing oscillator depends on the energy level and can be expressed explicitly in terms of the complete elliptic integral by variable substitutions in the energy integral,

$$
T=\frac{4}{\omega} K_{e}\left(m_{e}\right) .
$$

$K_{e}\left(m_{e}\right)$ is the complete elliptic integral [25], and the the parameters are are conveniently expressed in terms of the small-amplitude angular frequency $\omega_{0}=\sqrt{k / m}$ and the maximum amplitude $u_{m}$ as

$$
\omega=\omega_{0} \sqrt{1+\left(\lambda u_{m}\right)^{2}}, \quad m_{e}=\frac{\left(\lambda u_{m}\right)^{2}}{2\left(1+\left(\lambda u_{m}\right)^{2}\right)} .
$$


With the present displacement initial conditions $u_{m}=u_{0}$. At the amplitude level $u_{0}=1$ the period determined by the elliptic integral is $T=0.759 T_{0}=4.768$. The second order accuracy of the time integration is illustrated by the relative error on the numerically determined period $T_{n}$ in the form $\Delta T / T=\left(T_{n}-T\right) / T$ as a function of the non-dimensional time step $\omega_{0} h$. For a linear oscillator with angular frequency $\omega$ the period error in the equivalent linear numerical solution is given asymptotically by $\frac{1}{12}\left(\omega_{0} h\right)^{2}$. This relation is shown in Fig. 3 as a solid line while the results from the conservative time integration algorithm of Table I are marked by the + symbol.

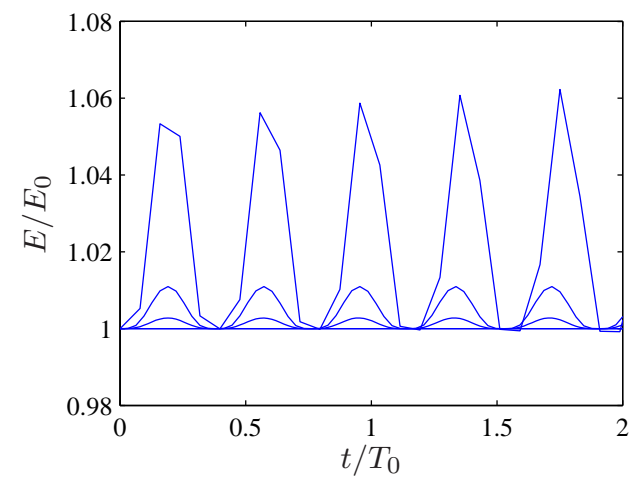

Figure 4. Duffing oscillator: Newmark total energy, $h=0.5,0.2,0.1$.

The equivalent solution by the average acceleration form of the Newmark algorithm with parameters $\gamma=2 \beta=\frac{1}{2}$ is obtained by setting $\Delta K=0$ in the present algorithm. Hereby the energy conservation property is lost as illustrated in Fig. 4, showing energy fluctuations $E(t) / E_{0}$ for $\omega_{0} h=0.5,0.2,0.1$ together with the constant energy level from the conservative algorithm. In the Newmark algorithm the period error $\Delta T / T$ and the magnitude of the energy fluctuation $\Delta E / E$ over the time history decrease proportional to $(\omega h)^{2}$, corresponding to quadratic convergence, as illustrated in Fig. 3. In a single-degree-of-freedom problem like the present, the effect of the energy fluctuations can fairly easily be reduced by decreasing the step size, but for multi-degree-of-freedom systems the effect of non-conservation may be quite significant due to sparse sampling of the high-frequency modes as illustrated in the elastic pendulum example in Section 5.3. In the limiting case of rigid constraints, the non-conserving property of the Newmark type algorithms constitutes a severe limitation on their use in multibody dynamics.

\subsection{Oscillator with hyperbolic sine stiffness}

The present example explores the algorithm in Table I in connection with an oscillator with the internal force represented by a sinh-function. The time integration can be performed at three levels: a simple Newmark type average acceleration form without specific account of the variable stiffness, a quartic approximation in which the stiffness increment is represented directly by $\Delta K$, and finally the fully conservative form in which the stiffness increment is represented by the secant form of $\mathbf{g}_{*}$ from (30).

The equation of motion has the generic form (48). In the present case the internal force and the internal energy are given in terms of the initial stiffness $k$ and the stiffness non-linearity 

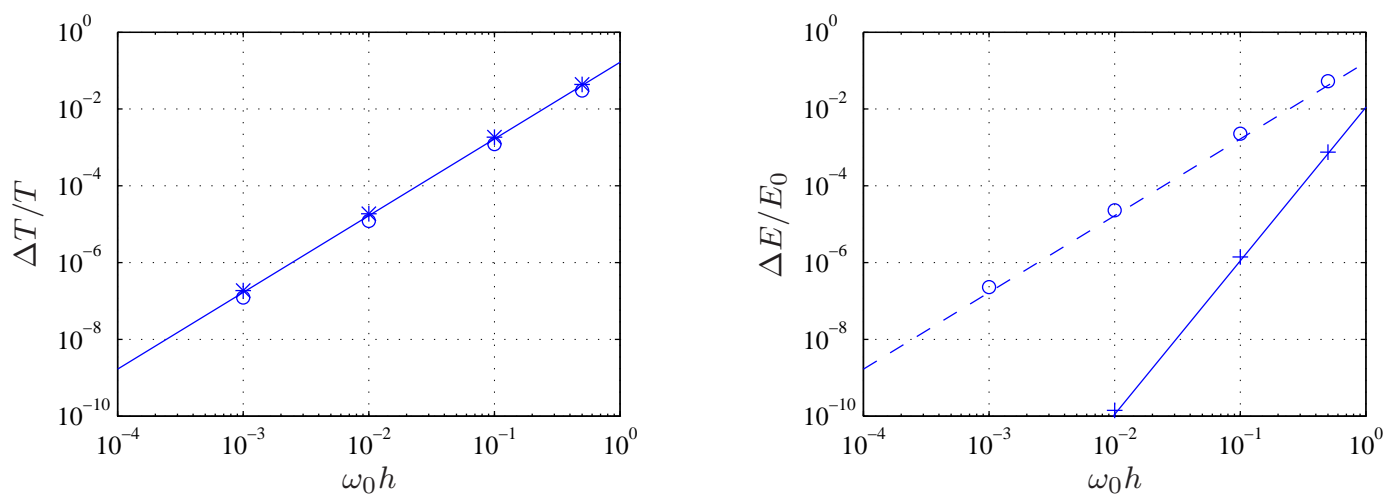

Figure 5. Hyperbolic oscillator $\lambda u_{m}=2.0$. a) Period error $\Delta T / T$, b) Energy error $\Delta E / E_{0}$. Conservative $\Delta K_{*}: \times$, Stiffness increment $\Delta K:+$, Newmark: $\circ$.

parameter $\lambda$ as

$$
g(u)=\frac{k}{\lambda} \sinh (\lambda u), \quad G(u)=\frac{k}{\lambda^{2}}(\cosh (\lambda u)-1) .
$$

The tangent stiffness follows by differentiation as

$$
K(u)=k \cosh (\lambda u) .
$$

As in the previous example $k=1$ and $m=1$. Taking $\lambda=2$ and the maximum displacement $u_{m}=1$ gives a relative increase of the tangent stiffness from equilibrium to maximum displacement of $\cosh (2)=3.762$, quite close to the relative stiffness increase of the Duffing oscillator in the previous example. The period of free oscillations follows from the procedure for the ideal pendulum, [26], by considering an imaginary parameter $i \lambda$. The result is expressed by the elliptic integral of the first kind (51) with the parameters

$$
\omega=\omega_{0} \cosh \left(\frac{1}{2} \lambda u_{m}\right), \quad m_{e}=\tanh ^{2}\left(\frac{1}{2} \lambda u_{m}\right) .
$$

In the present example $\lambda=2.0$, whereby the argument is $\frac{1}{2} \lambda u_{m}=1.0$. The period is $T=$ $0.796 T_{0}=4.999$ and the elliptic function parameter $m_{e}=0.5800$.

The response has been evaluated for $\omega_{0} h=0.5,0.1,0.01,0.001$ with three different forms of the algorithm: Newmark mid-point, stiffness increment $\Delta K$ corresponding to quartic energy, and fully conservative with secant representation of $\mathbf{g}_{*}$. The relative period error $\Delta T / T$ is shown in Fig. 5a together with the asymptotic estimate $\Delta T / T \simeq \frac{1}{12}\left(\omega_{0} h\right)^{2}$ for an equivalent linear oscillator with angular frequency $\omega_{0}$. The agreement is good and demonstrates the second order property of each of the three algorithms. The difference between the three algorithms is in the way they describe energy conservation. Figure $5 \mathrm{~b}$ shows the relative difference between maximum and minimum energy over a time of ten periods of oscillation. The Newmark average acceleration method is seen to be second order with respect to energy conservation, while the present method in the form with direct representation of the stiffness increment $\Delta K$ has fourth order energy conservation, and indeed a considerably smaller multiplicative constant. The final form based on inclusion of the secant representation of $\mathbf{g}_{*}$ conserves the energy to within a relative accuracy of $10^{-12}$ for the present set of tolerance parameters. The results suggest that for ordinary dynamics problems the form with direct use of the stiffness increment $\Delta K$ may 
be fully satisfactory, while full energy conservation to the accuracy of the computation can be obtained and may be needed for constrained problems.

\subsection{Elastic pendulum}

This example illustrates energy conservation and algorithmic dissipation by the elastic pendulum shown in Fig. 6. The pendulum consists of a concentrated mass $m$ suspended in a hinged elastic bar with negligible mass, stiffness $E A$, and length $l_{0}$ in the unloaded state. Gravitation gives the force $m g$ in the $x$-direction. The position of the mass is described by the coordinates $\mathbf{u}=[x, y]^{T}$ and the velocity is $\dot{\mathbf{u}}=[\dot{x}, \dot{y}]^{T}$. The kinetic and potential energy are

$$
T=\frac{1}{2} m\left(\dot{x}^{2}+\dot{y}^{2}\right), \quad U=\frac{1}{2} l_{0} E A \varepsilon^{2}-m g x,
$$

where $\varepsilon$ is the strain in the bar, corresponding to the force $N=E A \varepsilon$. In the present example the Green strain definition $\varepsilon=\left(l^{2}-l_{0}^{2}\right) / 2 l_{0}^{2}$ is chosen, whereby the energy potential is quartic in the displacement components. Thus, the effective internal force is represented by $\mathbf{g}_{\mathrm{q}}$ as given in (29) without use of the higher-order secant correction.

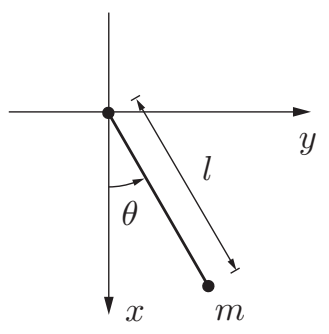

Figure 6. Elastic pendulum with concentrated mass.

The energy expressions define the mass matrix, the internal force, and the external force:

$$
\mathbf{M}=\left[\begin{array}{cc}
m & 0 \\
0 & m
\end{array}\right], \quad \mathbf{g}=\frac{N}{l_{0}}\left[\begin{array}{l}
x \\
y
\end{array}\right], \quad \mathbf{f}=\left[\begin{array}{c}
m g \\
0
\end{array}\right] .
$$

The tangent stiffness matrix $\mathbf{K}=\partial \mathbf{g} / \partial \mathbf{u}$ consists of a geometric and a constitutive part,

$$
\mathbf{K}=\frac{N}{l_{0}}\left[\begin{array}{ll}
1 & 0 \\
0 & 1
\end{array}\right]+\frac{E A}{l_{0}^{3}}\left[\begin{array}{ll}
x^{2} & x y \\
y x & y^{2}
\end{array}\right] .
$$

These expressions form the input to the conservative integration algorithm in Table I.

In the present example $m=1, l_{0}=1, g=10$ and $E A=3000$. The time scales of the problem are the period of pendulum vibrations $T_{p}=2 \pi / \omega_{p}=2 \pi / \sqrt{g / l_{0}}=1.987$ and the vibration period of the bar of $T_{b}=2 \pi / \omega_{b}=2 \pi / \sqrt{E A / m l_{0}}=0.1147$. The ratio of these time scales is $T_{p} / T_{b}=17.3$, and thus the oscillations of the bar are rapid relative to the swinging pendulum motion. The integration time increment is chosen as $h=0.02$, corresponding to about six points per period of the rapid axial vibrations of the bar. The initial conditions are zero velocity and $\left[x_{0}, y_{0}\right]=[0.0,1.1]$, corresponding to a $10 \%$ elongation of the bar. The change in potential energy in a similar rigid pendulum between the horizontal and vertical position is $m g l_{0}$. The additional internal elastic energy introduced by the initial stretch is $1.6537500 \mathrm{mgl}_{0}$, and thus there is a fair balance between the energy in the pendulum and axial vibration modes. 
The coordinates $x(t)$ and $y(t)$ are integrated with the conservative algorithm in Table I with $\varepsilon_{\mathbf{r}}=10^{-6} \mathrm{mg}$ and $\varepsilon_{\mathbf{u}}=10^{-6} l_{0}$, giving a maximum number of iterations of $i=4$. The representation of the contribution from the non-linear coupling terms in the approximate residual force stiffness matrix $\mathbf{K}_{*}$ in the form $\frac{1}{3} \Delta \mathbf{K}$, as derived from the integral mean property in Section 4.1, is superior to the ad hoc alternative of neglecting the contribution from the derivative of $\Delta \mathbf{K}$, leading to the alternative term $\frac{1}{6} \Delta \mathbf{K}$ of half the magnitude in the approximation of the residual force stiffness matrix. This choice leads to a maximum of $i=6$ iterations under the same conditions.
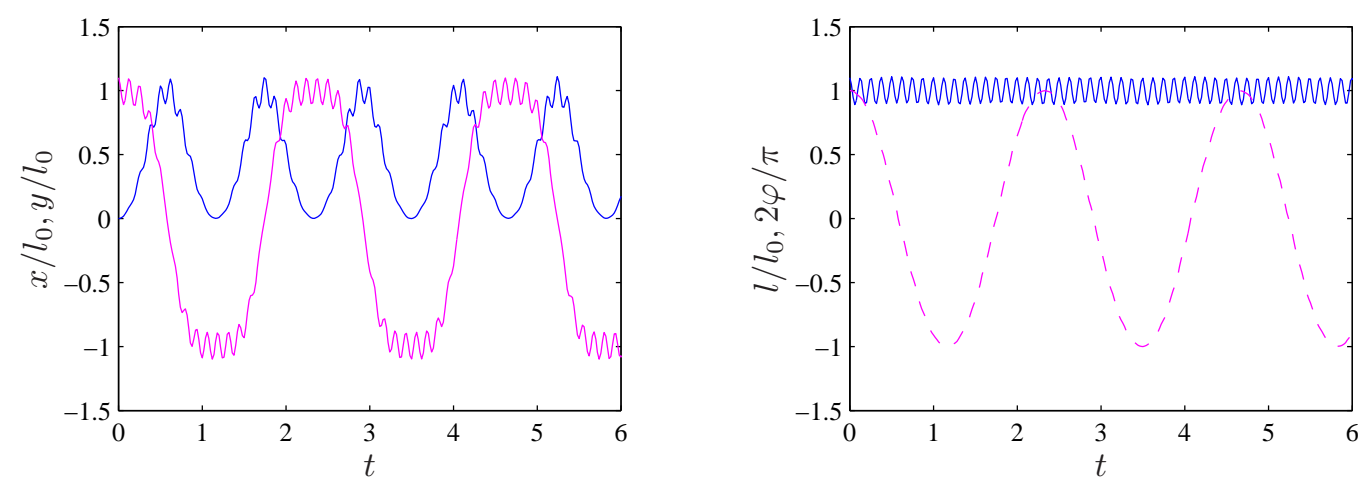

Figure 7. Pendulum with $h=0.03$. a) Coordinates $x(t)$ and $y(t)$, b) Length $l$ and angle $\varphi$.

The results for the position variables $x(t)$ and $y(t)$, used directly in the algorithm, are shown in Fig. 7a, while an alternative representation of the motion in terms of the length $l(t)$ and the angle $\varphi(t)$ is shown in Fig. 7b. Both figures clearly illustrate the slow pendulum motion superposed by the rapid oscillations of the elastic bar. The total energy of the pendulum is $E=U+T=1.6537500 \mathrm{mgl}_{0}$. The energy is conserved to within a relative error of $2 \cdot 10^{-8}$ with the present tolerances.

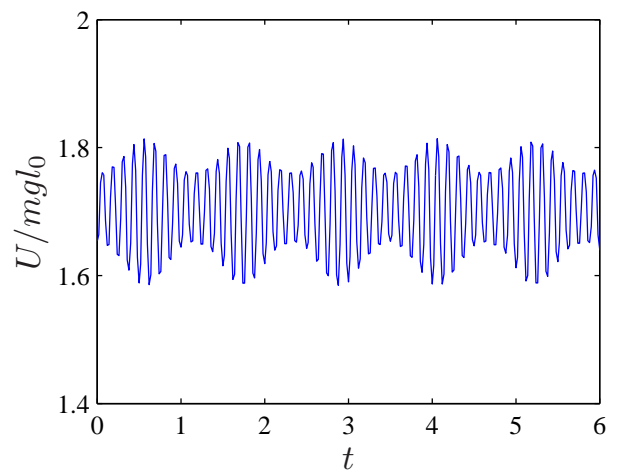

Figure 8. Energy of pendulum by Newmark algorithm with $\gamma=\frac{1}{2}, \beta=\frac{1}{4}$ and $h=0.02$.

The Newmark mean-acceleration algorithm with parameters $\gamma=2 \beta=\frac{1}{2}$ is obtained from the algorithm in Table I by imposing the condition $\Delta \mathbf{K} \equiv \mathbf{0}$. The energy time history for this algorithm is shown in Fig. 8 for $h=0.02$. It is seen that the omission of the $\Delta \mathbf{K}$ term leads to oscillations of the energy with a period corresponding to the bar vibrations, and amplitude varying in the interval $0.05-0.1 m g l_{0}$. As discussed in Example 1 it follows from the order of the $\Delta \mathbf{K}$ term in the algorithm that the energy error in the equivalent Newmark algorithm is 
proportional to $h^{2}$. In extreme cases this may lead to instability of the basic Newmark algorithm when used for nonlinear problems, a problem often countered by introducing extra algorithmic damping. Naturally, stabilization of the algorithm by algorithmic damping is undesirable, and even more so as the magnitude of the algorithmic damping needed for stabilization will typically not be known in advance.
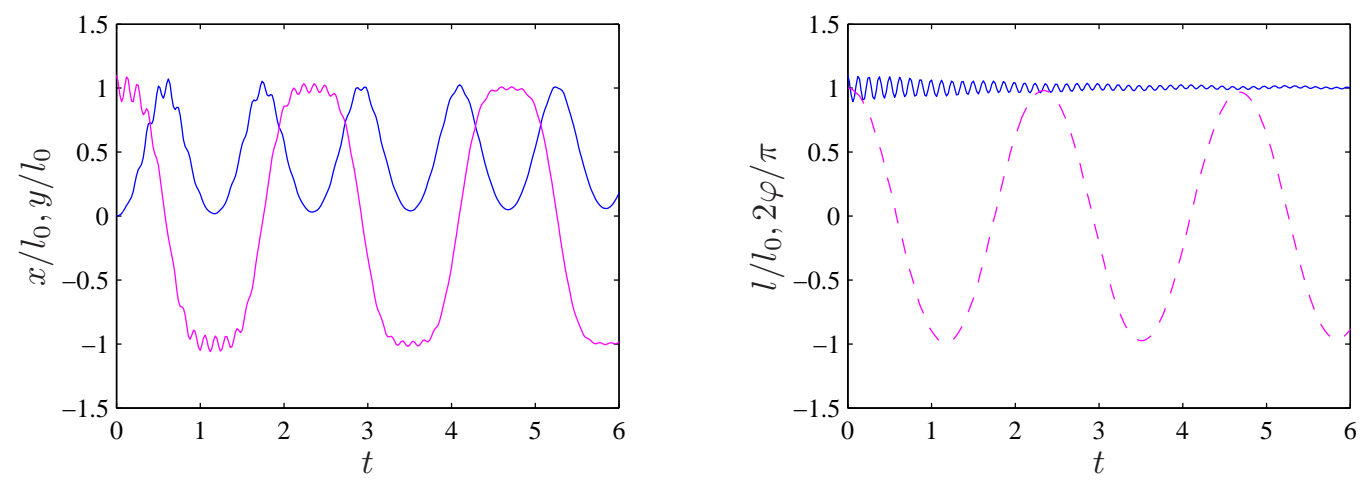

Figure 9. a) Coordinates $x(t)$ and $y(t), \mathrm{b})$ Length $l$ and angle $\varphi . h=0.02, \alpha=0.02$.

The present algorithm contains the option of introducing algorithmic damping to attenuate high-frequency components of the response. In practice the need for this type of algorithmic damping is typically associated with the use of interpolated elements, where the high-frequency components are not well represented by the polynomial shape functions. To illustrate the character of the simple algorithmic damping in terms of the parameter $\alpha$ the response of the pendulum is evaluated using the same parameters as before, but now with $\alpha=0.02$. By use of (18) it is found that this corresponds to introducing a damping ratio of magnitude $\zeta_{b} \simeq$ $\pi \alpha h / T_{b}=0.011$ in the bar vibration and $\zeta_{p} \simeq \pi \alpha h / T_{p}=0.0006$ in the pendulum vibration. The response is illustrated in Fig. 9. It is seen that while the high-frequency bar vibrations are damped out, the slow pendulum motion is left nearly undamped over the few periods shown. The energy development associated with the damped response is shown i Fig. 10. The zero level of the potential energy from gravity corresponds to $x=0$, i.e. horizontal position of the pendulum. It is observed that the bar vibration is nearly eliminated, and in addition a slow attenuation is introduced into the pendulum motion. After $t \simeq 4$ the pendulum does not retain enough energy to reach its horizontal position.

\section{CONCLUSIONS}

A single-step momentum and energy conserving time integration algorithm for systems with general internal energy function has been presented. It is derived in two steps: first for an internal energy of fourth degree in the displacements, and then generalized by a secant-type representation of the effective internal force to general energy potentials. The algorithm is second order accurate, but in addition conserves momentum and energy, making it unconditionally stable. In the case of a fourth degree internal energy function the energy conservation property is obtained by a representation of the effective internal force over the integration time interval in terms of the mean value of the internal forces at the interval endpoints plus an extra term containing the increment of the tangent stiffness matrix over the 


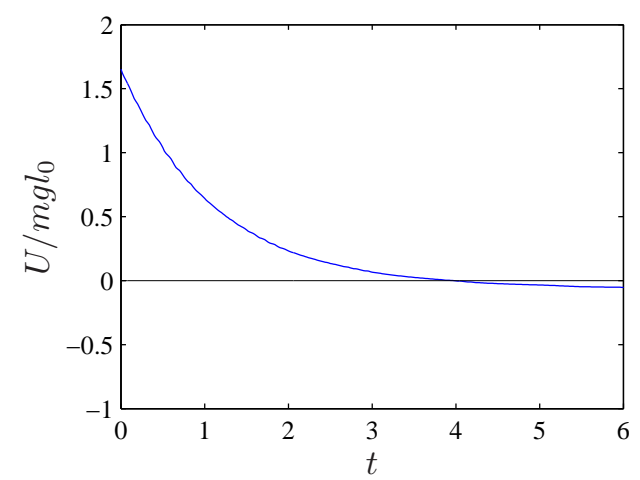

Figure 10. Energy decay of pendulum for $h=0.02$ and $\alpha=0.02$.

integration step. An approximate representation of the tangent stiffness of the algorithmic force has been derived in a form avoiding the use of derivatives of the stiffness matrix of the internal force. Typical iteration procedures make use of the tangent stiffness matrix anyway, and thus the present representation of the internal force does not introduce additional computations relative to typical collocation methods. Furthermore, this feature enables incorporation of this form of the algorithm into existing computer codes with minimal effort.

The general conservative form is obtained by introducing an additional fifth-order secant term that contains the increment of the internal energy. Thus, for systems with internal energy of order higher than four, full energy conservation requires the additional computation of the system energy in each iteration. This feature is easily incorporated into the algorithm via an 'if' statement, making the algorithm revert to the fourth order form, when this is appropriate.

A simple algorithmic damping option is included in the algorithm in terms of a single damping parameter. Essentially, it consists in a slight forward shift, when forming the mean values of the algorithm. The effect is somewhat similar to stiffness-proportional damping, but by including the same forward shift in the velocity representation, the algorithmic damping is without the phase dependence known from classic viscous damping, and furthermore increases monotonically with frequency without reduction at high frequencies due to aliasing.

\section{REFERENCES}

1. Newmark NM. A method of computation for structural dynamics. Journal of the Engineering Mechanics Division, ASCE 1959; 85:67-94.

2. Hilber HM, Hughes TJR, Taylor RL. Improved numerical dissipation for time integration algorithms in structural dynamics. Earthquake Engineering and Structural Dynamics 1977; 5:282-292.

3. Chung J, Hulbert GM. A time integration algorithm for structural dynamics with improved numerical dissipation: The generalized $\alpha$ method. Journal of Applied Mechanics 1993; 60:371-375.

4. Krenk S, Høgsberg JR. Properties of time integration with first order filter damping, International Journal for Numerical Methods in Engineering 2005; 64:547-566.

5. Hughes TJR, Analysis of transient algorithms with particular reference to stability behavior. In Computational Methods for Transient Analysis, Belytschko T, Hughes TJR (eds). North-Holland: Amsterdam, 1983; 67-155.

6. Krenk S. Energy conservation in Newmark based time integration algorithms. Computer Methods in Applied Mechanics and Engineering 2006; 195:6110-6124.

7. Hughes TJR, Caughy TK, Liu WK. Finite-element methods for nonlinear elastodynamics which conserve energy. Journal of Applied Mechanics 1978; 45:366-370. 
8. Kuhl D, Ramm E. Constraint energy momentum algorithm and its application to non-linear dynamics of shells. Computer Methods in Applied Mechanics and Engineering 1996; 136:293-315.

9. Kuhl D., Crisfield MA. Energy-conserving and decaying algorithms in non-linear structural dynamics. International Journal for Numerical Methods in Engineering 1999; 45:569-599.

10. Erlicher S, Bonaventura L, Bursi OS. The analysis of the generalized- $\alpha$ method for non-linear dynamics problems. Computational Mechanics 2002; 28:83-104.

11. Simo JC, Wong KK. Unconditionally stable algorithms for rigid body dynamics that exactly preserve energy and momentum. International Journal for Numerical Methods in Engineering 1991; 31:19-52.

12. Simo JC, Tarnow N. The discrete energy-momentum method. Conserving algorithms for nonlinear elastodynamics. Zeitschrift für angewandte Mathematik und Physik 1992; 43:757-792.

13. Romero I. An analysis of the stress formula for energy-momentum methods in nonlinear elastodynamics. Computational Mechanics 2012; 50:603-610.

14. Krenk S. The role of geometric stiffness in momentum and energy conserving time integration, International Journal for Numerical Methods in Engineering 2007; 71:631-651.

15. Gonzalez O. Time integration and discrete Hamiltonian systems. Journal of Nonlinear Science 1996; 6:449-467.

16. Gonzalez O. Exact energy and momentum conserving algorithms for general models in non-linear elasticity. Computer Methods in Applied Mechanics and Engineering 2000; 190:1763-1783.

17. Luenberger DG. Linear and Nonlinear Programming, 2nd ed., Addison-Wesley, Reading, Massachusets, 1989.

18. Greenspan D. Conservative numerical methods for $\ddot{x}=f(x)$. Journal of Computational Physics 1984; 56:28-41.

19. Betsch P, Steinmann P. Inherently energy conserving time finite elements for classical mechanics. Journal of Computational Physics 2000; 160: 88-116.

20. Betsch P, Steinmann P, Conservation properties of time FE method - Part II: Time-stepping schemes for non-linear elastodynamics, International Journal for Numerical Methods in Engineering 2001; 50:19311955.

21. Lens EV, Cardona A, Géradin M, Energy preserving time integration for constrained multibody systems, Multibody System Dynamics 2004; 11:41-64.

22. Celledoni E, Grimm V, McLachlan RI, McLaren DI, O'Neale D, Owren B, Quispel GRW, Preserving energy resp. dissipation in numerical PDEs using the 'Average Vector Field' method, Journal of Computational Physics 2012; 231:6770-6789.

23. Armero F, Romero I, On the formulation of high-frequency dissipative time-stepping algorithms for nonlinear dynamics. Part II: Second order methods, Computer Methods in Applied Mechanics and Engineering 2001; 190:6783-6824.

24. Krenk S. Non-linear Modeling and Analysis of Structures and Solids. Cambridge University Press, Cambridge, UK, 2009.

25. Abramowitz M, Stegun I. Handbook of Mathematical Functions, Dover, New York, 1965.

26. Symon KR, Mechanics, 2nd ed., Adison-Wesley, Reading, Massachusetts, 1967. 